\title{
Constraints on ultra-high-energy neutrino flux from radio observations of the Moon
}

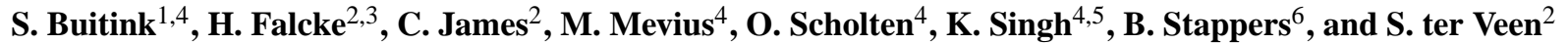 \\ ${ }^{1}$ Lawrence Berkeley National Laboratory, Berkeley, CA 94720, USA \\ ${ }^{2}$ Dept. of Astrophysics, IMAPP, Radboud University Nijmegen, 6500 GL Nijmegen, The Netherlands \\ ${ }^{3}$ ASTRON, P.O. Box 2, 7990 AA Dwingeloo, The Netherlands \\ ${ }^{4} \mathrm{KVI}$, University of Groningen, The Netherlands \\ ${ }^{5}$ Vrije Universiteit Brussel, Dienst ELEM, B-1050 Brussels, Belgium \\ ${ }^{6}$ Jodrell Bank Centre for Astrophysics, School of Physics and Astronomy, Univ. of Manchester, Manchester M13 9PL, UK
}

Correspondence to: S. Buitink (s.j.buitink@ rug.nl)

Received: 16 November 2010 - Revised: 30 June 2011 - Accepted: 21 September 2011 - Published: 22 August 2012

\begin{abstract}
The NuMoon project aims to study ultra-highenergy neutrinos and cosmic rays by using radio telescopes to search for short pulses from the Moon. These pulses are created when a neutrino or cosmic ray impinges on the Moon and interacts below the lunar surface. Part of the energy is converted into a hadronic shower, which emits radio emission in a process known as the Askaryan effect. In the first phase of the NuMoon project, $46 \mathrm{hrs}$ of data were collected with the Westerbork Synthesis Radio Telescope in a low frequency band: $40-80 \mathrm{MHz}$. This resulted in an upper limit on the neutrino flux above $10^{22} \mathrm{eV}$ which is an order of magnitude lower than previous limits. Additionally, an upper limit has been set on the ultra-high-energy cosmic-ray flux. The second phase of NuMoon will consist of observations with LOFAR.
\end{abstract}

\section{Introduction}

The origin of ultra-high-energy cosmic rays (UHE CRs) remains one of the big open questions in astrophysics. Modern experiments like the Pierre Auger Observatory (PAO) (Abraham et al., 2007) and HiRes (Abbasi et al., 2008) can efficiently detect UHE CRs, but there are two complications that make it hard to find their sources. First, the trajectories of CRs are bent in the (inter-)Galactic magnetic fields, so the arrival direction of the CRs at Earth does not align with the positions of their sources. This deflection depends on the charge of the CRs, and therefore on their composition. PAO has rejected CR isotropy above $6 \cdot 10^{19} \mathrm{eV}$ by studying a possible correlation with AGNs (Abraham et al., 2007). However, composition studies by PAO show a trend towards a heavier composition at the highest energies (Abraham et al., 2010), which suggests large separations between $\mathrm{CR}$ arrival directions and sources. In contrast, HiRes data does not reject isotropy but suggests a light CR composition (protons) (Abbasi et al., 2010). A second complication is that CRs above an energy of $4 \cdot 10^{19} \mathrm{eV}$ will interact with the cosmic microwave background (CMB). In these Greisen-Zatsepin-Kuzmin (GZK) interactions pions are created, which produce neutrinos when they decay (Greisen, 1966; Zatsepin and Kuzmin, 1966). The associated steepening of the CR spectrum has been confirmed experimentally (Abraham et al., 2008; Abbasi et al., 2008). The attenuation length of protons due to the GZK effect is $\sim 50 \mathrm{Mpc}$. Sources outside this radius cannot be found by studying UHE CRs directly. The detection of UHE neutrinos that are produced in GZK interactions or directly in the source is an attractive alternative to find these sources. Neutrinos are not affected by magnetic fields, and they can travel over cosmic distances almost unattenuated.

It was first suggested by Dagkesamanskii and Zheleznykh (1989) to use the Moon as a detector for UHE neutrinos and CRs. At energies above several PeV the Moon becomes opaque to neutrinos. Neutrinos impinging on the Moon will interact below the lunar surface. In a charged current interaction $\sim 20 \%$ of the energy is converted into a hadronic cascade. The leptons that are created are in principle detectable, though in the case of muons and taus the relevant cross-sections at UHE are highly uncertain, and their contribution is expected to be of order $10 \%$. Electrons initiate an electromagnetic shower which is hard to detect because it is elongated due to the LPM effect (Alvarez-Muñiz and Zas, 1998). However, at sufficiently high energy, the length and 


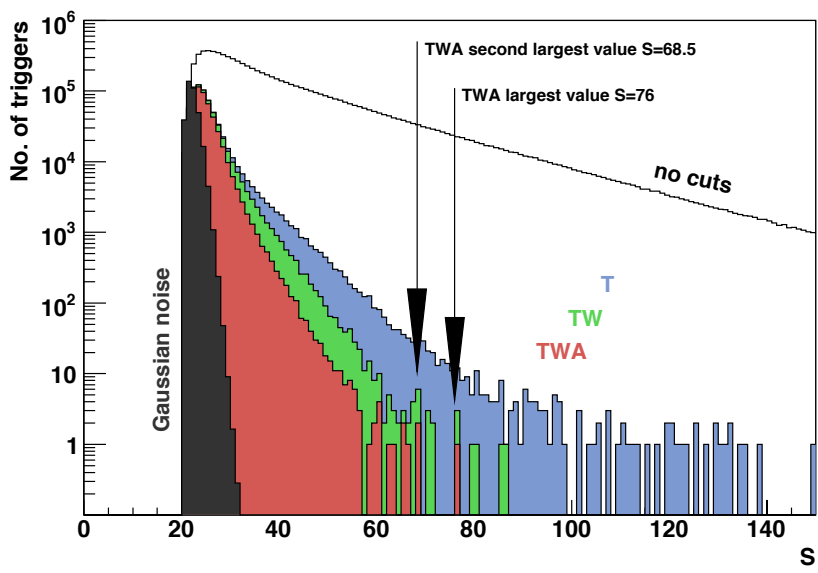

Fig. 1. Distribution of $S$ for several cuts. The top curve represents the distribution of raw triggers. The other distributions represent, in order of decreasing number of contained events, the T, TW, and TWA cut. The black area corresponds to the distribution of triggers that are expected for a background of pure Gaussian noise.

depth of the cascade is reduced because of the increase of the photonuclear and electronuclear cross sections (Gerhardt and Klein, 2010).

The cascade develops a negative charge excess propagating at the speed of light in vacuum, and produces radio emission (Askaryan, 1962). Although the radiation is most intense at the Cherenkov angle, it can be misleading to think of it in terms of pure Cherenkov radiation. The rise and decay of a moving charge itself would also produce radiation if the index of refraction of the medium were unity (ter Veen et al., 2010; James et al., 2010a). Actually, at low frequencies, when the wavelength is larger than the cascade length, the radiation is coherent at angles far from the Cherenkov angle, and emitted almost isotropically (Zas et al., 1992). This is exactly the reason why the NuMoon experiment uses a low frequency band (Scholten et al., 2006). The isotropic component of the radiation is more likely to escape the Moon. For example, if a cascade is directed parallel to or away from the lunar surface, radiation at the Cherenkov angle does not escape the Moon due to internal reflection. Therefore, at high frequencies, only cascades directed towards the surface can be detected at Earth. Such cascades only occur at the rim of the Moon, when neutrinos enter from behind (Gorham et al., 2004). At low frequencies, the isotropic radiation is able to escape the Moon for most geometries and the complete visible lunar surface is part of the effective detector volume.

GLUE (Gorham et al., 2004) was one of the pioneering experiments at high frequencies $(>1 \mathrm{GHz})$. Presently, LUNASKA performs high frequency measurements with the Parkes radio telescope. The restricted range in neutrino arrival directions that produce a signal at Earth allows for directional flux limits (James et al., 2010b). RESUN (Jaeger et al., 2010) performs $1.4 \mathrm{GHz}$ observations with the Ex- panded Very Large Array. The NuMoon project uses a low frequency bandwidth which offers an optimal detection probability at the highest energies. In the first phase, observations were performed with the Westerbork Synthesis Radio Telescope (WSRT). In these proceedings, we present the results of this phase (Scholten et al., 2009) and discuss the prospects of the second phase which employs LOFAR.

\section{NuMoon phase I: WSRT}

In 2007 and 2008 a total of $46.7 \mathrm{hrs}$ of data have been collected with the WSRT, an array telescope consisting of 14 parabolic $25 \mathrm{~m}$ telescopes on a $2.7 \mathrm{~km}$ east-west line, of which eleven were used in this experiment. The NuMoon experiment uses the Low Frequency Front Ends (LFFEs) which cover the frequency range $115-180 \mathrm{MHz}$. Each LFFE records full polarization data. The Pulsar Machine II (PuMa II) backend (Karuppusamy et al., 2008) can record a maximum bandwidth of $160 \mathrm{MHz}$, sampled as 8 subbands of $20 \mathrm{MHz}$ each. For each subband, the time series data is recorded at several storage nodes with a sampling frequency of $40 \mathrm{MHz}$. The signals of the eleven radio dishes are merged to form two beams in different directions, aimed at opposite sides of the Moon. Each beam uses 4 subbands.

The raw data is transformed into the frequency domain in order to identify strong sources of man-made Radio Frequency Interference (RFI). These sources show up as strong narrow lines in the frequency spectrum, and are filtered out by flagging and removing the affected frequencies from the band.

Next, the data is compensated for dispersion in the Earth's ionosphere. The dispersion causes an offset between pulses in different frequency bands as well as a broadening of the pulses themselves. The amount of dispersion depends on the Total Electron Content (TEC) of the ionosphere at the time of measurement, and the elevation of the Moon. For example, for a typical TEC value of ten, a subband pulse is broadened by $\sim 200 \mathrm{~ns}$. Because of variations in the thickness of the ionosphere on short timescales and over distance, we assume the presence of an error in the TEC value used for de-dispersion.

After de-dispersion, the data is transformed back into the time-domain to search for pulses. Most of the power of a bandwidth limited, Nyquist sampled pulse with a small amount of dispersion is contained within 5 time bins. We define $P_{5}$ as the power integrated over 5 consecutive samples normalized over one trace

$P_{5}=\frac{\sum_{5 \text { samples }} P_{x}}{\left\langle\sum_{5 \text { samples }} P_{x}\right\rangle}+\frac{\sum_{5 \text { samples }} P_{y}}{\left\langle\sum_{5 \text { samples }} P_{y}\right\rangle}$,

where the averaging is done over one time trace (20000 time samples), and $x$ and $y$ denote the two polarizations. Because 


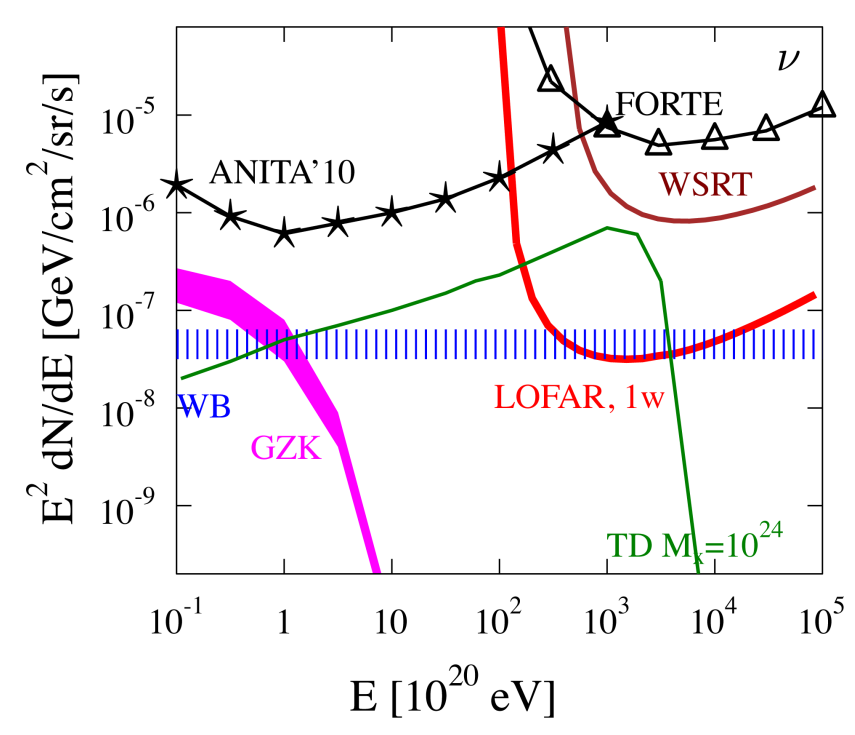

Fig. 2. Neutrino flux limit currently established with $46.7 \mathrm{hrs}$ of WSRT data. Limits set by ANITA (Gorham et al., 2009) and FORTE (Lehtinen et al., 2004) are included in the plot as well as the Waxman-Bahcall flux (Waxman and Bahcall, 1998) and a TD model prediction (Protheroe and Stanev, 1996). The band shows the systematic error.

RFI has been removed at this point the background is expected to be fairly constant over time. All measurements have been done at times when the Moon was not near the Galactic plane or the horizon. The trigger condition is set to be $P_{5}>5$ for all four frequency subbands. The value $S$ is defined as the sum over the maximum $P_{5}$ values in the four frequency bands.

Figure 1 shows the distribution of $S$ for triggered events. Several cuts are applied. First, at regular intervals, strong pulses were found with a technical origin. The affected time intervals were cut out resulting in a $\sim 10 \%$ loss of observation time. Secondly, the width of the pulses, defined as the number of consecutive $P_{5}$ values that exceed threshold should be less than 12 in all subbands. The third condition is that a proper lunar pulse should be visible in only one of the two beams.

Figure 1 shows the distribution of $S$ of triggered events at various cut levels: time interval cut $(\mathrm{T})$, time and width cut (TW) and all cuts including anti-coincidence (TWA). The line enclosing the black area corresponds to the distribution of triggers that are expected if the background is pure Gaussian noise. After all cuts have been applied the number of triggers is a factor of $3-4$ higher than the amount of triggers expected for Gaussian noise. Apparently, the background includes pulsed noise that produces triggers that survive the cuts.

The efficiency with which pulses are found by the analysis procedure and the effects of data cuts and ionospheric dispersion are simulated by adding pulses to raw data. This

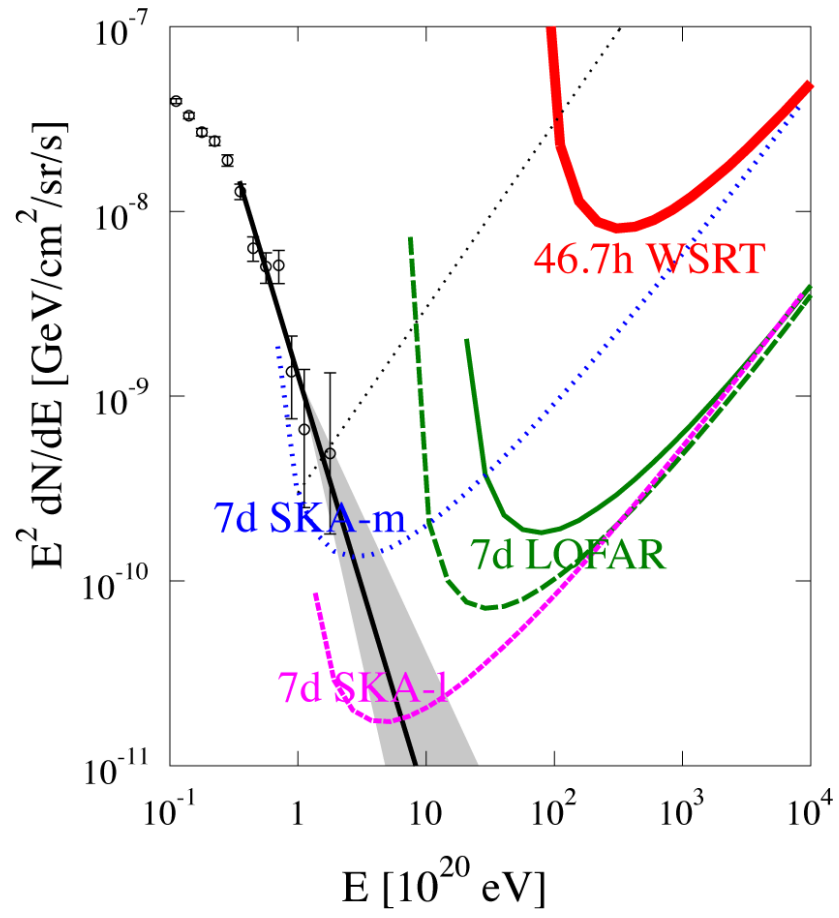

Fig. 3. CR upper limit with $90 \%$ confidence level from WSRT measurements and sensitivities of LOFAR and SKA. See text for details.

results in a detection efficiency (DE) which depends on the strength of the input pulses. The effects of Faraday rotation in the ionosphere is included in the calculation.

In $46.7 \mathrm{hrs}$ of data no triggers were found with a strength exceeding $S_{t h}=77$. From simulation we find that pulses in excess of $S_{i}>120$ are detected with a DE of $87.5 \%$. This pulse strength corresponds to $240 \mathrm{kJy}$.

The lack of pulses stronger than a certain magnitude implies a new limit on the flux of UHE neutrinos. Obtaining the limit requires a calculation of the acceptance which takes into account the attenuation of the radio signal inside the Moon, the transmission at the lunar surface and the angle with respect to the arrival direction of the neutrino. On basis of the simulations which are described in Scholten et al. (2006), the $90 \%$ confidence level flux limit has been determined (see Fig. 2). Further details are found in Buitink et al. (2010).

With the same data a limit can be set on the UHE CR flux (Fig. 3). A cascade induced by a CR will be initiated just below the lunar surface in contrast to neutrino induced showers. Therefore, the radio signal suffers less attenuation. However, it also raises the question whether the radiative field can properly develop in a volume that is smaller than the wavelength of the radiation. A detailed derivation (ter Veen et al., 2010; James et al., 2010a) shows that such a formation zone is not needed. This becomes evident when one realizes that a system of growing and decaying current would also radiate if it were to develop in a vacuum. Hence, the dielectric medium is not essential for the formation of the 
radiative field, although it certainly affects it.

\section{NuMoon phase II: LOFAR}

The next phase in the NuMoon experiment will be to use LOFAR, the Low Frequency Array (Falcke et al., 2006), that is under construction in the Netherlands and several other European countries. LOFAR is a network of thousands of low frequency omni-directional radio antennas communicating over a fiber optics network. The signals of individual antennas are phased electronically to form multiple simultaneous beams on the sky. NuMoon will use the high band LOFAR antennas $(110-240 \mathrm{MHz})$. The very large baselines make it possible to form beams much smaller than the size of the Moon. It is therefore possible to localize precisely the origin of a radio pulse. The predicted sensitivity of 1 week of data from the dense LOFAR core is included in Fig. 2.

Figure 3 shows the expected sensitivity of LOFAR to the UHE CR flux, as well as the $90 \%$ confidence level limit established with the WSRT measurements. The black data points are from PAO measurements Abraham et al. (2008). The solid black line is an extension of this flux with a power law of index -4.3 (grey band corresponds to 0.8 uncertainty). The dotted black line is the formal modelindependent differential flux limit based on PAO results.

The Square Kilometer Array (SKA ${ }^{1}$ ) will reach the sensitivity needed to detect CRs around and above the GZK cutoff. It will offer a broad frequency range, that allows for observations in different windows. The low band $(100-300 \mathrm{MHz})$ offers the best sensitivity at higher energies, while the intermediate band $(300-500 \mathrm{MHz})$ will be sensitive to lower energies and offers the possibility of directional reconstruction, since the radiation at higher frequencies is emitted close to the Cherenkov angle.

\section{Conclusion}

We have derived a limit on the UHE neutrino flux using WSRT observations of the Moon. Unprecedented sensitivity could be reached because the observation frequency is much lower than other lunar Cherenkov experiments. With the increased sensitivity of next-generation radio arrays like LOFAR and SKA, lunar radio observations can potentially become an important tool in neutrino astrophysics.

Acknowledgement.

Edited by: K.-H. Kampert

Reviewed by: two anonymous referees

\footnotetext{
${ }^{1}$ http: //www.skatelescope.org
}

\section{References}

Abbasi, R. U. et al. (IceCube collaboration): First Observation of the Greisen-Zatsepin-Kuzmin Suppression, Phys. Rev. Lett., 100, 101101, 2008.

Abbasi, R. U. et al. (IceCube collaboration): Indications of ProtonDominated Cosmic-Ray Composition above 1.6 EeV, Phys. Rev. Lett., 104, 161101, 2010.

Abraham, J. et al. (Pierre Aguer collaboration): Correlation of the Highest-Energy Cosmic Rays with Nearby Extragalactic Objects, Science, 318, 938, 2007.

Abraham, J. et al. (Pierre Aguer collaboration): Observation of the Suppression of the Flux of Cosmic Rays above $4 \times 10^{19} \mathrm{eV}$, Phys. Rev. Lett., 101, 061101, 2008.

Abraham, J. et al. (Pierre Aguer collaboration): Measurement of the Depth of Maximum of Extensive Air Showers above $10^{18} \mathrm{eV}$, Phys. Rev. Lett., 104, 091101, 2010.

Alvarez-Muñiz, J. and Zas, E.: The LPM effect for EeV hadronic showers in ice: implications for radio detection of neutrinos, Phys. Lett. B, 434, 396, 1998.

Askaryan, G.: Excess Negative Charge of an Electron-Photon Shower And Its Coherent Radio Emission, Sov. Phys. JETP, 14, 441, 1962.

Buitink, S. et al. (NuMoon collaboration): Constraints on the flux of ultra-high energy neutrinos from Westerbork Synthesis Radio Telescope observations, Astronom. Astrophys., 521, A47+, 2010.

Dagkesamanskii, R. D. and Zheleznykh, I. M.: A radio astronomy method of detecting neutrinos and other superhigh-energy elementary particles, Pis ma Zhurnal Eksperimental noi i Teoreticheskoi Fiziki, 50, 233, 1989.

Falcke, H. D. E. et al. (LOFAR collaboration): LOFAR- The Low Frequency Array, Long Wavelength Astrophysics, 26th IAU meeting, Joint Discussion 12, 21 August 2006, Prague, Czech Republic, JD12, 16, 12, 2006.

Gerhardt, L. and Klein, S. R.: Electron and photon interactions in the regime of strong Landau-Pomeranchuk-Migdal suppression, Phys. Rev. D, 82, 074017, 2010.

Gorham, P. W. et al. (ANITA collaboration): Experimental Limit on the Cosmic Diffuse Ultrahigh Energy Neutrino Flux, Phys. Rev. Lett., 93, 41101, 2004.

Gorham, P. W. et al. (ANITA collaboration): New Limits on the Ultrahigh Energy Cosmic Neutrino Flux from the ANITA Experiment, Phys. Rev. Lett., 103, 051103, 2009.

Greisen, K.: End to the Cosmic-Ray Spectrum?, Phys. Rev. Lett., 16, 748, 1966.

Jaeger, T. R., Mutel, R. L., and Gayley, K. G.: Project RESUN, a Radio EVLA Search for UHE Neutrinos, Astropart. Phys., 34, 293, 2010.

James, C. W. et al.: General description of electromagnetic radiation processes based on instantaneous charge acceleration in “endpoints", Phys. Rev. E, 84, 056602, 2010a.

James, C. W. et al.: LUNASKA experiment observational limits on UHE neutrinos from Centaurus A and the Galactic Centre, M0n. Not. Roy. Astron. Soc., 1425, 2010b.

Karuppusamy, R., Stappers, B., and Van Straten, W.: PuMa-II: A Wide Band Pulsar Machine for the Westerbork Synthesis Radio Telescope, Publ. Astron. Soc. Pacific, 120, 191, 2008.

Lehtinen, H. et al.: FORTE satellite constraints on ultrahigh energy cosmic particle fluxes, Phys. Rev. D, 69, 013008, 2004. 
Protheroe, R. and Stanev, T.: Limits on Models of the Ultrahigh Energy Cosmic Rays Based on Topological Defects, Phys. Rev. Lett., 77, 3708, 1996.

Scholten, O. et al. (NuMoon collaboration): Optimal radio window for the detection of Ultra-High Energy cosmic rays and neutrinos off the moon, Astropart. Phys., 26, 219, 2006.

Scholten, O. et al. (NuMoon collaboration): Improved flux limits for neutrinos with energies above $10^{22} \mathrm{eV}$ from observations with the Westerbork Synthesis Radio Telescope, Phys. Rev. Lett., 103, 191301, 2009. ter Veen, S. et al. (NuMoon collaboration): Limit on the ultrahighenergy cosmic-ray flux with the Westerbork synthesis radio telescope, Phys. Rev. D, 82, 103014, 2010.

Waxman, E. and Bahcall, J. N.: High energy neutrinos from astrophysical sources: An upper bound, Phys. Rev., D59, 023002, 1998.

Zas, E., Halzen, F., and Stanev, T.: Electromagnetic pulses from high-energy showers: Implications for neutrino detection, Phys. Rev. D, 45, 362, 1992.

Zatsepin, G. T. and Kuzmin, V. A.: Upper limit of the spectrum of cosmic rays, JETP Lett., 4, 78, 1966. 